\title{
The 3C Chandra snapshot survey
}

\author{
Alessandro Paggi ${ }^{1}$, F. Massaro ${ }^{2}$ and D. E. Harris ${ }^{1}$ \\ ${ }^{1}$ Harvard-Smithsonian Center for Astrophysics \\ 60 Garden Street, Cambridge, MA 02138, USA \\ email: apaggi@cfa.harvard.edu \\ ${ }^{2}$ SLAC - National Laboratory and Kavli Institute for Particle Astrophysics and Cosmology \\ 2575 Sand Hill Road, Menlo Park, CA 94025, USA
}

\begin{abstract}
We present a uniform analysis of Chandra archival observations of a complete sample of $\sim 2003 \mathrm{C}$ sources at $z<0.5$. We measured the $\mathrm{X}$-ray intensity of the nuclei and of any radio hot spots and jet features with associated X-ray emission. X-ray fluxes in three energy bands, i.e., soft, medium, and hard, for all the sources analyzed are also reported. For the stronger nuclei, we also applied the standard spectral analysis, which provides the best-fit values of the $\mathrm{X}$-ray spectral index and absorbing column density. In addition, a detailed analysis of bright $\mathrm{X}$-ray nuclei that could be affected by pile-up has been performed. X-ray emission was detected for all the nuclei of the radio sources in our sample.
\end{abstract}

Keywords. galaxies: active, BL Lacertae objects: general, galaxies: jets, radio continuum: galaxies, X-rays: galaxies

\section{Main results}

We find a sizable fraction $(\sim 30 \%$ at $z<0.3, \sim 60 \%$ at $0.3<z<0.5)$ of our sources showing evidence of significant absorption $\left(N_{H}>5 \times 10^{22} \mathrm{~cm}^{-2}\right)$. In particular, for 3C105, we analyzed the archival observations performed by XMM-Newton and Swift and we found that its core is absorbed (in agreement with our Chandra spectral analysis). We also found a marginal detection of FeK emission line and evidence of a soft X-ray excess. Among our 3C Chandra observations, we detected three CSS radio sources (3C93.1, 3C258 and 3C67), one WAT radio galaxy (3C89 hosted in the cluster of galaxies with the largest known X-ray cavity) and X-ray emission from galaxy clusters surrounding 3C196.1, 3C19 and 3C320 (Figs. 1 and 2). We find X-ray emission from nine radio hotspots, several knots in the radio jets of 3C327.1 and 3C341, and in the case of 3C459 we find emission coincident with the eastern radio lobe. Finally, we find X-ray emission cospatial with two radio jets (3C29 and 3C402), and in the FR II radio galaxies 3C187 and 3C313 we discovered extended X-ray emission still of unknown nature arising from regions along the principle radio axis.

\section{References}

Gilbert, G. M., Riley, J. M., Hardcastle, M. J., et al. 2004, MNRAS, 351, 845

Hardcastle, M. J., Evans, D. A., \& Croston, J. H. 2009, MNRAS, 396, 1929

Hardcastle, M. J., Massaro, F., \& Harris, D. E. 2010, MNRAS, 401, 2697

Massaro, F., Harris, D. E., Tremblay, G. R., et al. 2010, ApJ, 714, 589

Massaro, F., Tremblay, G. R., Harris, D. E., et al. 2012, ApJS, 203, 31

Massaro, F., Harris, D. E., Tremblay, G. R., et al. 2013, ApJS, 206, 7

Spinrad, H., Marr, J., Aguilar, L., \& Djorgovski, S. 1985, PASP, 97, 932 


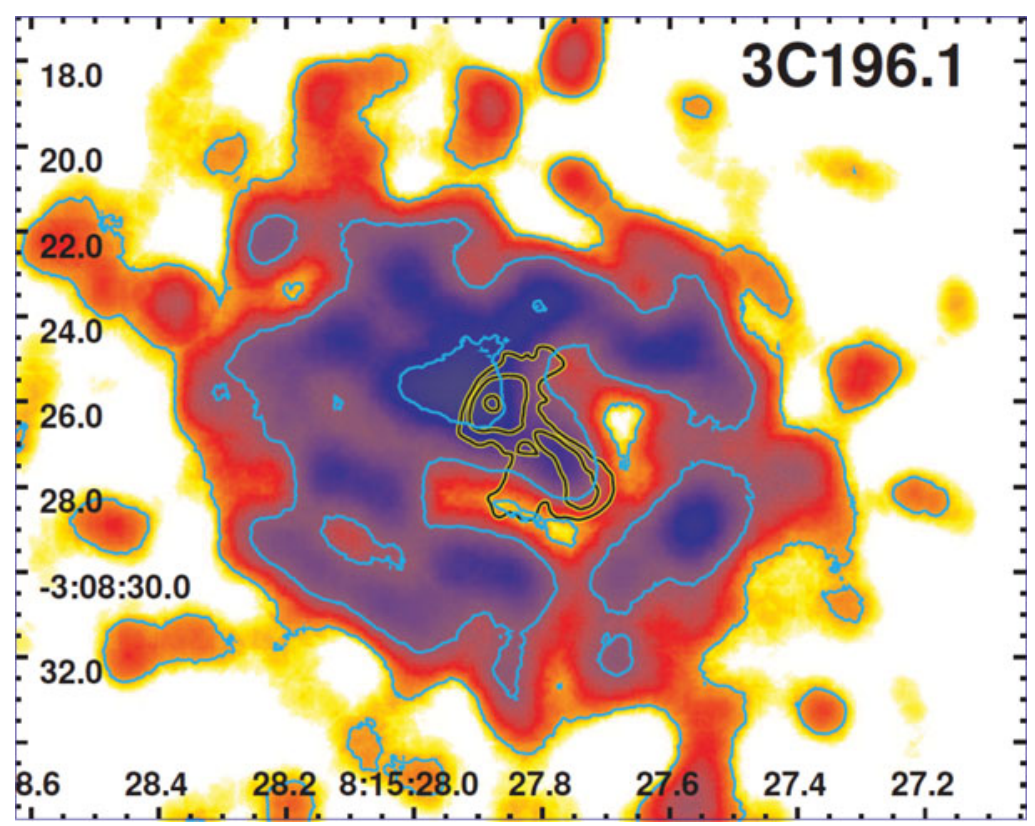

Figure 1. 3C196.1 Chandra image $(0.5-7 \mathrm{keV})$ with a pixel scale of 0 ".492 and smoothed with a Gaussian of FWHM $=11$ ". X-ray contours are presented in cyan while radio $8.4 \mathrm{GHz}$ VLA contours are presented in black.

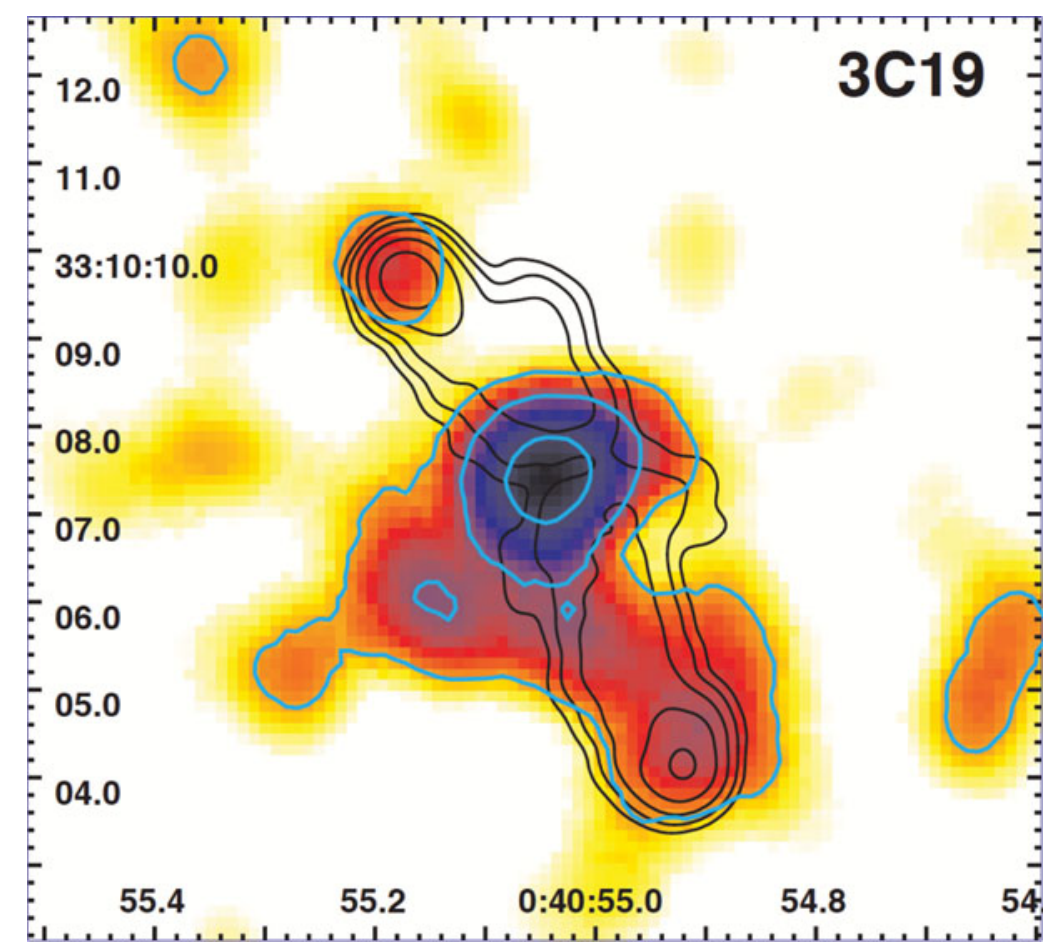

Figure 2. 3C19 Chandra image $(0.5-7 \mathrm{keV})$ with a pixel scale of 0 ".123 and smoothed with a Gaussian of FWHM $=1$ ".3. X-ray contours are presented in cyan while radio $4.86 \mathrm{GHz} \mathrm{VLA}$ contours are presented in black (courtesy of M. Hardcastle; Gilbert et al. 2004). 